\title{
A longitudinal study of dominance and aggression in greylag geese (Anser anser)
}

\author{
Brigitte M. Weiß, ${ }^{a, b}$ Kurt Kotrschal,, a,c and Katharina Foerster ${ }^{a, d}$ \\ ${ }^{a}$ Department of Behavioural Ecology, Institute of Biology, University of Neuchâtel, Rue Emile Argand 11, \\ 2000 Neuchâtel, Switzerland, ' Konrad Lorenz Forschungsstelle, Fischerau 11, 4645 Grünau im Almtal, \\ Austria, ${ }^{\mathrm{C} D e p a r t m e n t}$ of Behavioural Biology, University of Vienna, Althanstraße 14, 1090 Vienna, \\ Austria, and ${ }^{\mathrm{d}}$ Comparative Zoology, Institute of Evolution and Ecology, University of Tübingen, Auf der \\ Morgenstelle 28, 72076 Tübingen, Germany
}

\begin{abstract}
A variety of factors are known to affect dominance and aggression in social vertebrates. In the present study, we used a long-term data set on greylag geese (Anser anser) to investigate the complex relationships between individual life histories, the social environment, and dominance-related behaviors. We applied a multifactorial approach to assess the relative importance of factors in different life-history stages. Previous studies in geese documented effects of sex and social status and achieved differing results for the effects of family size, age, and body weight on dominance and aggression. Extrinsic factors like season or flock structure were generally not considered. Our analyses showed that a considerable number of factors related to individual life histories, season, and the social environment affected dominance and aggression in greylag geese, but not all significant effects were necessarily strong effects. Pronounced effects on aggression rates were caused by the flock's sex ratio, parental effects, individual social status, and sex. Whether individuals interacted with the same opponents repeatedly was influenced most by parental effects and the sex ratio, whereas the strongest determinants of dominance rank were parental effects and social status. Hence, dominance behaviors may not only be influenced by intrinsic factors but also by season and an individual's social environment. Furthermore, our study indicates that optimal choices for achieving or maintaining a high dominance rank may vary considerably between life-history stages. This highlights the value of long-term studies and multifactorial approaches for understanding the complexities of dominance relationships in social vertebrates. Key words: agonistic interactions, dominance hierarchy, effect size, flock structure, parental effects, social status. [Behav Ecol 22:616-624 (2011)]
\end{abstract}

\section{INTRODUCTION}

$\mathrm{D}$ ominance and aggression play an important role in many animal societies. Dominance and related behaviors may enhance access to limited resources (e.g., Ficken et al. 1990; Prop and Deerenberg 1991; Stahl et al. 2001), thereby enhancing survival (e.g., Kikkawa 1980; Arcese and Smith 1985; Stahl et al. 2001) and reproductive success (e.g., Dunbar 1980; Kikkawa and Wilson 1983; Black and Owen 1987; Pusey et al. 1997). However, high levels of aggression and achieving or maintaining a high dominance rank may also be energetically costly, may increase risk of injury, or may be stressful for an individual involved in numerous agonistic interactions (hereafter social stress, e.g., Hogstad 1987; Kotrschal et al. 1998; Sapolsky 2005). Hence, individuals need to optimize their behavioral investment according to their own condition as well as to their environment.

The suite of factors known to affect dominance and aggression in social vertebrates varies considerably between species, ranging from mainly size-determined dominance rank in fish (e.g., Buston 2003) to maternally transmitted rank in many primates and hyenas ("maternal rank inheritance," e.g., Dunbar 1980; Horrocks and Hunte 1983; Engh et al. 2000). In birds, dominance and aggression are known to be affected by

Address correspondence to B.W. Weiß, who is now at Institute of Evolution and Ecology, Auf der Morgenstelle 28, D-72076 Tübingen, Germany. E-mail: brigitte.weiss@klf.ac.at.

Received 26 July 2010; revised 6 February 2011; accepted 9 February 2011

(c) The Author 2011. Published by Oxford University Press on behalf of the International Society for Behavioral Ecology. All rights reserved.

For permissions, please e-mail: journals.permissions@oup.com a variety of different factors, including sex (e.g., Brown 1963; Arcese and Smith 1985; Kikkawa et al. 1986; Kotrschal et al. 1993), age (e.g., Arcese and Smith 1985; Estevez et al. 2003), and genetic predisposition (Craig et al. 1965; Boag 1982). In addition to these intrinsic factors, some studies also showed influences of environmental factors such as season (Tarvin and Woolfenden 1997), habitat (Gregoire and Ankney 1990), and food availability (Kotrschal et al. 1993) as well as influences of the social environment, for example, sex composition of the sibling group (Boag and Alway 1980, but see Arcese and Smith 1985) and group size (Estevez et al. 2003). Influential factors may predict which individuals will achieve and ultimately benefit from a high dominance rank (Richner 1989) and may thereby enhance our understanding of the functional roles of dominance and aggression. However, although a multitude of influential factors have been identified, interdependencies between factors as well as their relative importance remain little understood. A thorough assessment of such problems may require individual-based long-term studies spanning the full life histories of a large number of individuals, which provide reliable estimates and thereby offer unique insights into the causes and complexities of animal behavior and ecology (Clutton-Brock and Sheldon 2010).

Here, we present data from a longitudinal study on greylag geese (Anser anser). Geese are highly social birds (Kotrschal et al. 2010) that form large flocks for most of the year and form long-term monogamous pair bonds. In most species, including greylag geese, young stay with their parents throughout an entire year (primary families) and sometimes rejoin their parents for a second year (secondary families). Mixed species flocks may occur, whereby interspecific 
encounters seem to be determined mostly by the species' body size, maintenance of family bonds, and the number of individuals per species present (Madsen 1985; Kristiansen and Jarrett 2002; Jónsson and Afton 2008). For intraspecific interactions, it is well documented that males are more aggressive and more dominant than females (e.g., Boyd 1953; Black and Owen 1987; Poisbleau et al. 2006) and that families dominate pairs in aggressive encounters, whereas pairs tend to win against single individuals (e.g., Boyd 1953; Raveling 1970; Lamprecht 1986; Gregoire and Ankney 1990; Kotrschal et al. 1993; Poisbleau et al. 2006). Agonistic interactions within pairs or families are extremely rare (Boyd 1953; Scheiber et al. 2009a), and members of a social unit actively and passively support each other in conflicts with other flock members (Weiß and Kotrschal 2004; Scheiber et al. 2005, 2009a, 2009b). Several studies further showed that family size affected rates of aggression as well as dominance rank (whitefronted geese, A. albifrons, Boyd 1953; snow geese, A. caerulescens, Gregoire and Ankney 1990; barnacle geese, Branta leucopsis, Loonen et al. 1999), but such effects were absent in other studies (bar-headed geese, A. indicus, Lamprecht 1986; brent geese, B. bernicla, Poisbleau et al. 2006; snow geese, Mulder et al. 1995). Differing results were also obtained about the effects of individual body size and age (e.g., barnacle geese: Black and Owen 1989; Stahl et al. 2001, bar-headed geese: Lamprecht 1986, brent geese: Poisbleau et al. 2006). Cross-fostering experiments in barnacle geese suggested that parental effects also contribute to rank acquisition in juveniles through both learning and heredity (Black and Owen 1987), and levels of aggression varied depending on habitat (snow geese: Gregoire and Ankney 1990) and food availability (greylag geese: Kotrschal et al. 1993, snow geese: Mulder et al. 1995).

Although the effects of sex and social status (single, paired, or family) in particular have been assessed thoroughly in geese, extrinsic factors like the social environment remain little understood. However, agonistic interactions depend not only on an individual's own behavior but also on that of its conspecifics, and consequently, the social environment may be expected to play a major role in dominance and aggression. In addition, only a few studies have attempted to assess the relative importance of several influential factors (Lamprecht 1986; Black and Owen 1989). Furthermore, due to the migratory behavior of many goose populations, studies were often limited to certain seasons and lifehistory stages, but as individual needs change throughout the year and life history, optimal behavior and influences thereupon are also likely to change throughout life. Over 15 years of observations on the dominance structure of a flock of greylag geese provide the framework for a longitudinal and multifactorial approach, where we used repeated measures over the individuals' lifetime to investigate parental effects and effects of individual life histories, seasonal variation, and flock characteristics. In particular, we aimed to perform a comprehensive analysis of dominance in greylag geese by 1) assessing which intrinsic and extrinsic factors influence dominance rank and related behaviors, 2) determining the relative importance of influential factors, and 3) comparing effects across the main life-history stages, thereby taking into consideration factors particular to the respective life-history stages, such as family structure or pair-bond characteristics.

\section{MATERIALS AND METHODS}

\section{Animals}

A flock of greylag geese was introduced into the valley of the river Alm, Austria, by Konrad Lorenz and coworkers in 1973
(Lorenz 1988). Like numerous other flocks of greylag geese, the flock is nonmigratory, and due to the secluded location of the valley, mixing with other greylag geese is nearly absent. The geese are unrestrained and roam the valley between the Konrad Lorenz Research Station and a lake $10 \mathrm{~km}$ to the south, where they roost at night. All birds are individually marked with colored leg bands after capture by hand or in a walk-in trap $(\sim 3 \times 2 \times 2 \mathrm{~m})$ baited with grains and food pellets. Individual life histories have been closely monitored since the establishment of the flock. Over the years, flock size varied between 110 and 180 individuals. As in other populations, natural predation, mainly by red foxes (Vulpes vulpes), is common and may account the loss of up to $10 \%$ of the adult flock per year (Hemetsberger 2001). The flock is well habituated to the presence of humans and is supplemented with food pellets and grains twice a day. Food is distributed by research station staff on the meadows around the research station in low quantities from spring to fall and in sustaining amounts during winter. About $25 \%$ of the individuals were hand raised by human foster parents, employing a well-established hand-raising tradition. Goslings thereby are accompanied by their foster parents for $24 \mathrm{~h}$ per day from hatching to fledging and follow the spatiotemporal patterns of the goose families (Hemetsberger et al. 2010). Hand-raised individuals maintain a life-long confidence toward familiar humans but typically treat unfamiliar humans with the same cautiousness as the goose-raised birds. They are indistinguishable from their goose-raised conspecifics in most life-history aspects, for example, breeding parameters, reproductive success, age of first pair bond, or pair-bond duration (Hemetsberger et al. 2010).

\section{Data collection}

Data on the dominance structure of the flock were collected during 41 observation periods of 5 days each between winter 1995 and summer 2009. Each year, data were collected during late summer, following reestablishment of the flock after molt (beginning of August to beginning of October), during midwinter when the flock was stable (mid-December to beginning of January), and during the mating season in February. Because of continuous harsh weather conditions in winter 2000, the flock frequently remained at the lake used as their night roost. There, geese were typically spread out over the expanse of water, and reliable individual identification was not possible; hence, no data were collected in winter 2000. From the onset of the breeding season in March to the end of wing molt in July, the flock disintegrates and spreads out over the entire valley, and so, no data were collected in those months. The study thus covers behavior outside the breeding season.

In each 5-day observation period, agonistic interactions were observed by scan sampling the flock prior to and during feedings, when most or all flock members were assembled around the feeding area. Hence, during these times, flock members of all ages and status classes had the opportunity to interact with each other, whereas at other times of the day, the geese were spread out over the valley, and observations of agonistic interactions might have been biased by the composition (sex, age, social status, and individual identity) of the observed subgroup. An agonistic interaction was defined as an encounter between 2 geese in which one of them evoked withdrawal from the other (e.g., Kristiansen and Jarrett 2002; Jónsson and Afton 2008). For all observed agonistic interactions, the identities of the winner and loser were determined, whereby the withdrawing goose was considered the loser of the interaction and the goose that evoked the withdrawal was considered the winner. Comparable with other studies (e.g., Appleby 1983; Gregoire and Ankney 1990; Côté 2000), the winner was the initiator of the interaction in the vast majority of interactions $(>99 \%$, Weiß, BM, unpublished 
observations). We thus did not distinguish further between initiated and won interactions. Data collection was terminated when more than one third of the flock had left the feeding area, which was typically 10-30 min after feeding, depending on the season. In addition to agonistic interactions, time at onset and duration of the observation, weather conditions and presence or absence of each flock member were recorded for each observation session. The life-history parameters of each individual (age, current social status, and pair-bond duration) and flock structure were recorded at the end of the 5-day observation period. A complete list of parameters is provided in Supplementary Material, Supplementary Table S1. All data were collected by B.M.W. with the exception of summer 2000 , when data were collected by another experienced observer following the above protocol.

\section{Data analysis}

A median of 1554 (range: 747-2150) agonistic interactions were recorded per observation period, equaling a median of 21 (range: 1-189) interactions per individual per observation period. For each observation period, we calculated individual aggression rates as the number of won agonistic interactions per hour. To distinguish between geese that were aggressive against other flock members in general and geese that repeatedly attacked only few opponents, we calculated an index of specificity as the number of individuals a bird interacted with out of the total number of that bird's interactions. Hence, a value of 1 would indicate low specificity, that is, that an individual had different opponents in all its interactions, whereas a value close to 0 would be assigned to an individual that only interacted with one particular opponent and thus showed high specificity. Finally, dominance rank was calculated as the number of individuals defeated out of the total number of individuals interacted with. For this measure, we only considered individuals that had been observed in an agonistic interaction at least 5 times.

We conducted generalized linear mixed models (GLMMs) using the GenStat 12.1 statistical package, applying the restricted maximum likelihood procedure for repeated sampling with an unbalanced design. GLMMs were constructed with aggression rate, specificity, or dominance rank as the response variable and individual identity as random term to account for repeated measurements (1-41 observations per individual, mean \pm standard error: $13.7 \pm 11.5)$. For each response variable, we calculated a main model comprising the whole data set $(n=6108$ observations from a total of 445 different individuals), with a poisson error distribution for aggression rate and a binomial error distribution for specificity and dominance rank. Model fit was assessed by inspecting whether the residuals from the final model showed a nearrandom distribution and by evaluating the dispersion parameter. The set of fixed terms included parental effects, lifehistory effects, seasonal effects, flock structure (see Supplementary Material, Supplementary Table S1 for a full list and description of fixed terms) and some first order interactions (see results Figure 1). To facilitate interpretation of interactions and to be able to investigate effects applicable only to certain life-history stages, we constructed further GLMMs for 5 major life-history stages: juveniles $(<1$ year old), subadults ( 1 to $<2$ years old), single adults, paired adults, and parental birds, whereby all adults were 2 years of age or older (see Supplementary Material, Supplementary Table S2 for numbers of observations and individuals). Stage-specific GLMMs were constructed in a similar manner as the main models, whereby the set of fixed terms was adjusted to each life-history stage and included stage-specific terms such as pair-bond or family characteristics (see Supplementary Material, Supplementary Table S1). In the "RESULTS" section, we report results of the main model and major deviances from and additions to the main model for the different life-history stages. Details of the models for the various life-history stages are described in the Supplementary Material.

We sequentially deleted fixed terms in order of decreasing significance, starting with any interaction terms in the model (Galwey 2006; Garamszegi et al. 2009). Only terms with $P<0.1$ (main term) or $P<0.05$ (interaction term) remained in the final model. Main terms with $0.05<P<0.1$ were kept in the model to account for tendencies; however, only terms with $P<$ 0.05 were considered as significant effects. To account for nonlinear effects of age, we also modeled the quadratic effects of age $\left(\right.$ age $\left.^{2}\right)$. Therefore, whenever "age $\mathrm{e}^{2 "}$ remained in the model, the linear term "age" was kept in the model even if it was not significant. Excluded terms were reentered one by one into the final model to confirm that these terms did not explain a significant part of the variation (Galwey 2006; Poesel et al. 2006). To obtain $P$ values for main terms involved in an interaction, we calculated the same model without the interaction term but took all other statistical information from the interaction model. To evaluate the relative importance of terms in the final model, we compared their effect sizes (Garamszegi et al. 2009), whereby the effect size of a factor was considered to be the range of effect sizes (minimum to maximum) across the factor levels.

\section{RESULTS}

\section{Aggression rate}

\section{Main model}

The number of attacks per hour was significantly influenced by parental effects, life history (except for the raising history), season, and flock structure (Table 1), whereby the strength of the effects varied considerably (Figure 1). In particular, identity of the parents had a very strong effect on individual aggression. Effects of sex and social status were also pronounced: In line with previous studies, males were more aggressive than females, and families were more aggressive than pairs, which were more aggressive than single individuals (Figure 2). Other life-history parameters (e.g., age) had significant but very small effects on aggression rates.

Aggression rates were highest during the mating season and lowest during winter, but generally, the season only had a moderate effect. Notably, the sex ratio in the flock had a very large effect: Individuals were more aggressive, the more males the flock contained. In addition to these main effects, we found complex interactions between sex, status, and other main effects (Figure 1 and see life-history stages).

\section{Life-history stages}

Deviations from the general pattern as well as more specific effects were found in the analysis of the different life-history stages. This particularly concerned parental effects, which were large in the main model but were absent in adult singles and parental birds. Life-history effects were generally similar across models, with the exception of the raising history. Unlike in the main model, raising significantly influenced aggression rates of juveniles and subadults. Interestingly, gooseraised juveniles were more aggressive than hand-raised ones, whereas this effect was reversed in subadults. Seasonal effects were of moderate size in all models, but life-history stages differed in who was most aggressive at what time of year. The main difference between models concerning flock structure was an absence of sex ratio effects in subadults. In addition, 2 parameters specific to particular life-history stages had pronounced effects on aggression rates. In paired adults, pairbond type affected aggression: Heterosexual pairs were most aggressive and homosexually paired males least aggressive. 


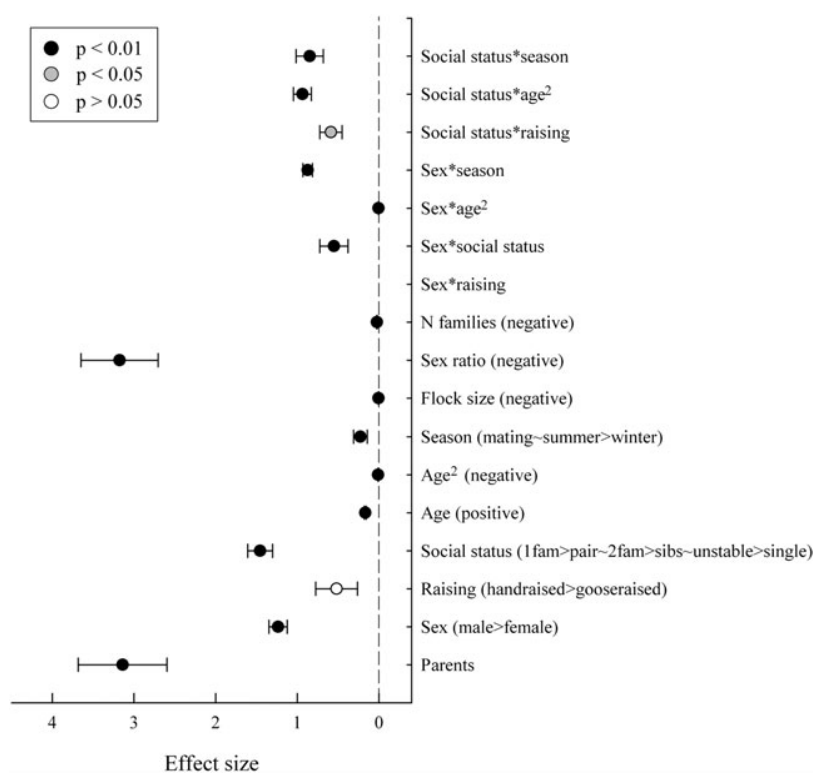

Figure 1

Effect sizes \pm standard error of parameters influencing aggression rates in greylag geese. All tested terms and interactions of terms are given on the $y$ axis. A description of the effect is given in brackets for the main effects. Missing values indicate terms that were nonsignificant and were removed from the full model. "1fam" = primary family, "2fam" = secondary family, and "sibs" = sibling group.

Furthermore, family type affected aggression in parental birds: Parents of primary families were more aggressive than those of secondary families (see Supplementary Material, Supplementary Table S2 and Figures S1a-S5a).

\section{Specificity}

Main model

The specificity of agonistic interactions was influenced by all investigated parameters except the raising history and the number of families in the flock (Table 1). Again, effect sizes varied considerably between the parameters (Figure 3), with parents having the largest effect on specificity. Males were also more specific in their interactions than females, and socially unstable individuals (e.g., courting or challenged individuals) were the most specific social class, but in general, life-history effects were not pronounced. Similarly, season only had a moderate effect, whereby specificity was highest during the mating season and lowest in winter. The only flock characteristic with a pronounced effect was the sex ratio of the flock: Specificity was higher at times with a more male-biased sex ratio. Furthermore, effects of sex, status, age, and season significantly interacted with each other (Figure 3 and see life-history stages).

\section{Life-history stages}

Parental effects were large in the main model, yet parental effects did not significantly influence specificity in all life-history stages. Although parents significantly affected specificity in juveniles and paired adults, this pattern was absent in subadults, adult singles, and parental birds. Life-history effects were generally similar between the main model and the lifehistory stages; however, sex differences were absent in juveniles and status differences were absent in subadults. Seasonal effects showed no major differences between the models. Finally, as in the main model, sex ratio had a large effect in most life-history stages but did not significantly influence juvenile specificity. Pair-bond type and family type had moderate effects on the specificity of paired adults and parental birds, respectively, but none of the other stage-specific terms had noteworthy effects on specificity (see Supplementary Material, Supplementary Table S2 and Figures S1b-S5b).

\section{Dominance rank}

\section{Main model}

In general, dominance rank was influenced by all intrinsic parameters, by season and by the number of families in the flock but not by flock size or sex ratio (Table 1). However, effect sizes of significant terms varied considerably, with the largest effect being due to the identity of the parents (Figure 4). Males were more dominant than females, and families

Table 1

Fixed effects of parents, life-history traits, season, and flock structure on aggression rate, specificity, and dominance rank in greylag geese $(N=6108$ observations in 445 individuals $)$

\begin{tabular}{|c|c|c|c|c|c|c|c|c|c|c|c|c|c|}
\hline Fixed effects & df & \multicolumn{4}{|c|}{ Aggression rate } & \multicolumn{4}{|c|}{ Specificity } & \multicolumn{4}{|c|}{ Dominance rank } \\
\hline Parents & 75 & 3.139 & 0.544 & 133.8 & $<0.001$ & 8.081 & 0.965 & 102.2 & 0.039 & 3.42 & 0.607 & 131.8 & $<0.001$ \\
\hline Raising & 1 & 0.519 & 0.256 & 3.57 & 0.06 & & & & & 0.926 & 0.292 & 8.72 & 0.003 \\
\hline Social status & 5 & 1.455 & 0.152 & 1565.9 & $<0.001$ & 0.525 & 0.123 & 214.7 & $<0.001$ & 2.228 & 0.154 & 2721.0 & $<0.001$ \\
\hline Age & 1 & 0.167 & 0.01 & 267.2 & $<0.001$ & -0.071 & 0.009 & 61.9 & $<0.001$ & 0.136 & 0.011 & 169.0 & $<0.001$ \\
\hline Flock size & 1 & -0.004 & 0.001 & 36.77 & $<0.001$ & 0.001 & 0.001 & 4.31 & 0.038 & & & & \\
\hline Sex ratio & 1 & -3.177 & 0.473 & 45.11 & $<0.001$ & 2.359 & 0.42 & 31.58 & $<0.001$ & & & & \\
\hline$n$ families & 1 & -0.024 & 0.003 & 51.17 & $<0.001$ & & & & & -0.042 & 0.004 & 129.33 & $<0.001$ \\
\hline Sex $\times$ raising & 1 & & & & & & & & & -0.278 & 0.129 & 4.62 & 0.032 \\
\hline Sex $\times$ social status & 5 & -0.55 & 0.173 & 23.66 & $<0.001$ & 0.479 & 0.125 & 26.64 & $<0.001$ & 0.696 & 0.149 & 33.98 & $<0.001$ \\
\hline Sex $\times$ age $^{2}$ & 1 & -0.004 & 0.001 & 28.12 & $<0.001$ & & & & & -0.004 & 0.001 & 31.31 & $<0.001$ \\
\hline Sex $\times$ season & 2 & 0.872 & 0.059 & 234.8 & $<0.001$ & -0.378 & 0.053 & 60.5 & $<0.001$ & 0.461 & 0.058 & 64.6 & $<0.001$ \\
\hline
\end{tabular}

Individual identity was included as a random factor in all models. Effects are only shown for terms that remained in the final model. Wald and $p$ values in bold were determined from models without interaction terms. "Effect" $=$ effect size; " $n$ families" $=$ number of families. 


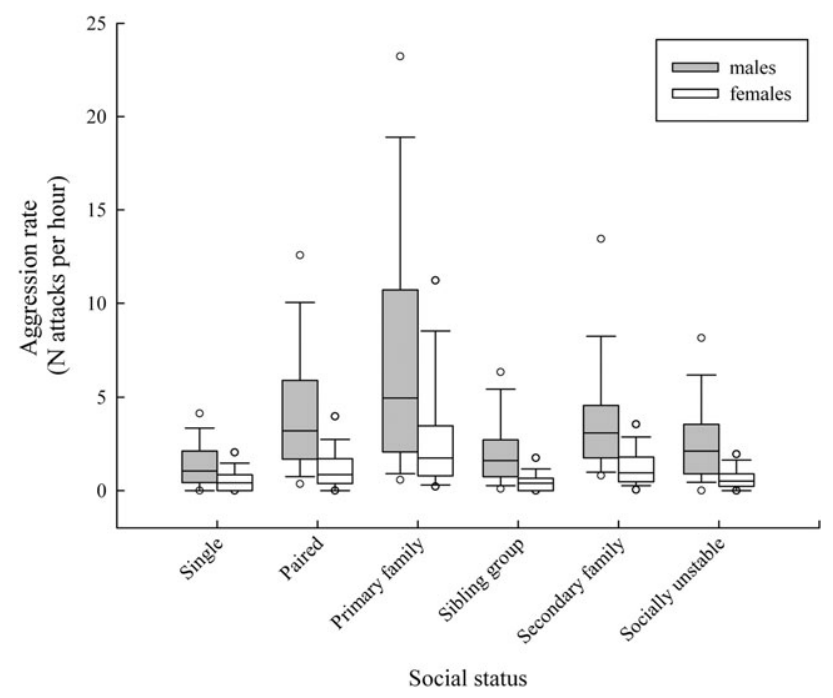

Figure 2

Status differences in aggression rates of male (gray) and female (white) greylag geese. Boxplots show medians and quartiles, whiskers 10 th and 90th percentiles, and circles 5th and 95th percentiles.

were dominant over pairs and singles, and social status had the second strongest effect on dominance rank (Figure 5). Unlike aggression rate or specificity, the raising history also influenced dominance rank, with hand-raised individuals generally ranking higher than goose-raised individuals.

Geese defeated fewer of their opponents during the mating season than during the rest of the year, but once again, seasonal effects were only of moderate size. Flock structure had only small or no effects on dominance rank. The effects of sex and social status further interacted with age, raising, and seasonal effects in a complex manner (Figure 5, see also lifehistory stages).

\section{Life-history stages}

As for the other investigated behaviors, parental effects were not apparent in all life-history stages. In particular, parental effects were absent in adult singles but were pronounced, albeit not significantly, in the other adult life-history stages. Males were always higher ranking than females, but sex was a very strong determinant in parental birds and had a rather small effect on singles. Most notably, the raising history had very diverse effects on dominance rank. Although hand-raised individuals were generally higher ranking than goose-raised ones, the exact opposite was true in juveniles (Figure 6). In adult birds, hand-raised individuals were only marginally higher ranking than goose-raised ones, and differences were not significant. Seasonal effects were small to moderate. However, as already observed for aggression rates, life-history stages differed considerably with respect to the season in which they achieved the highest success in agonistic interactions. Flock structure had no overall effect on dominance rank, but it did affect juveniles and subadults. Interestingly, dominance rank of juveniles increased as the rate of females in the flock decreased, whereas this effect was once again reversed in subadults. Most of the investigated stage-specific parameters had only small or no effects on dominance rank, with the exception of pair-bond type and family type. Comparable with aggression rates, heterosexually paired individuals were higher ranking than homosexually paired ones, and primary families were dominant over secondary families (Supplementary Material, Supplementary Table S2 and Figure S1c-5c).

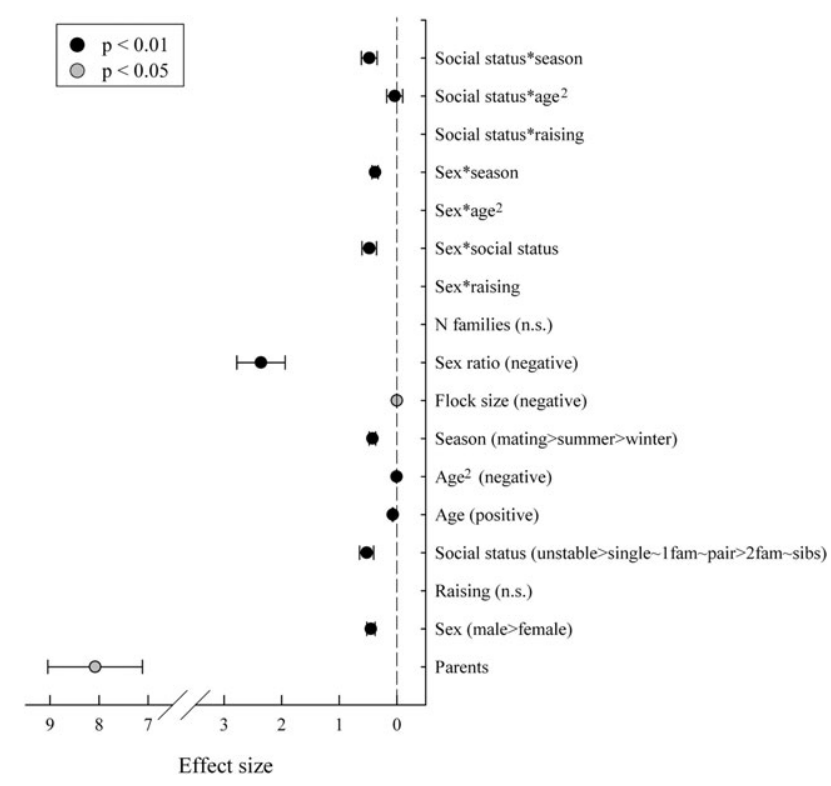

Figure 3

Effect sizes \pm standard error of parameters influencing specificity in greylag geese. All tested terms and interactions of terms are given on the $y$ axis. A description of the effect is given in brackets for the main effects. Missing values indicate terms that were nonsignificant and were removed from the full model. " 1 fam" = primary family,

"2fam" = secondary family, "sibs" = sibling group, and "n.s." = not significant.

\section{DISCUSSION}

The present study showed that aggressive behavior and dominance rank in greylag geese were influenced by a variety of factors, including parental effects, life-history effects, season, and flock structure. However, although a large number of

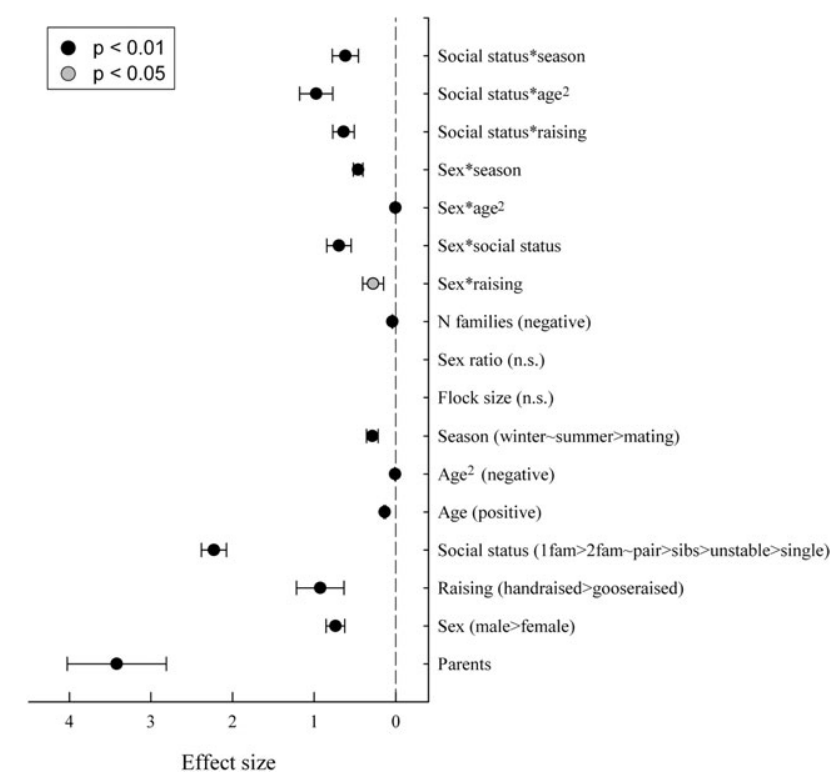

Figure 4

Effect sizes \pm standard error of parameters influencing dominance rank in greylag geese. All tested terms and interactions of terms are given on the $y$ axis. A description of the effect is given in brackets for the main effects. Missing values indicate terms that were nonsignificant and were removed from the full model. "1fam" = primary family, "2fam" = secondary family, "sibs" = sibling group, and "n.s." = not significant. 


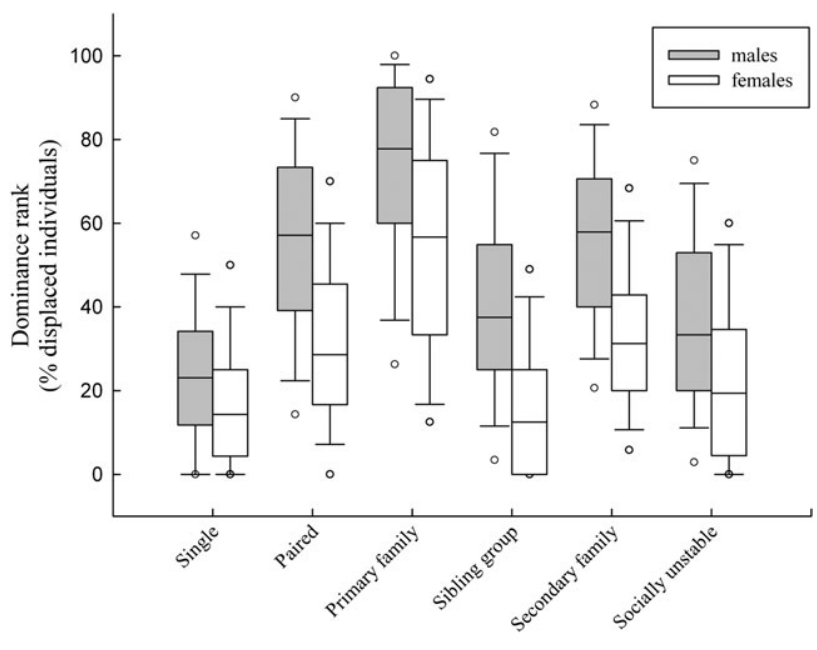

Social status

Figure 5

Status differences in dominance rank of male (gray) and female (white) greylag geese. Boxplots show medians and quartiles, whiskers 10th and 90th percentiles, and circles 5th and 95th percentiles.

factors were found to have significant effects, effect sizes varied considerably, and several factors, although significant, had only very small effects on the investigated behaviors. Our study thus confirmed, complemented, and realigned the importance of factors previously known to affect dominance and aggressive behavior in geese. The most pronounced determinants of aggression rates were the sex ratio of the flock and parental effects as well as social status and sex of the individual. Variation in specificity was considerably smaller than in the other behaviors, and the only strong effects on specificity were those of parents and sex ratio. Dominance rank was strongly influenced by parents and social status and, to a lesser extent, by sex and the raising history. This general picture differed throughout the different life-history stages. The differences particularly concerned effects of parents, raising history, season, and sex ratio and reflect changes in the

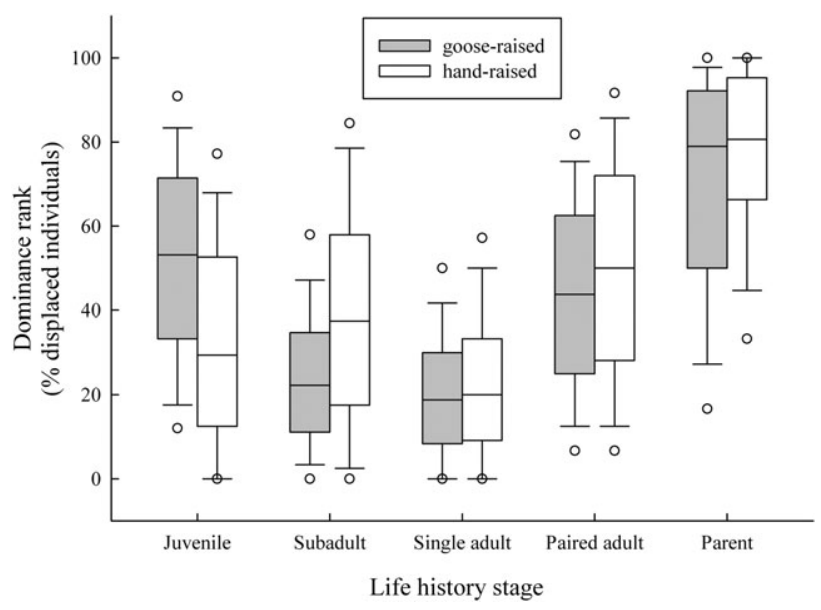

Figure 6

Dominance rank in goose-raised (gray) and hand-raised (white) geese in the 5 main life-history stages. Boxplots show medians and quartiles, whiskers 10th and 90th percentiles, and circles 5th and 95th percentiles. functional significance as well as social constraints during different times of the year and life.

\section{Parental effects}

In our study, parental effects were among the strongest determinants of all investigated behaviors. This complements the results of a cross-fostering study in juvenile barnacle geese: Black and Owen (1987) showed that young from dominant parents ranked highest, young from subordinate parents raised by dominant parents ranked intermediate, and those from subordinate parents ranked lowest, thus suggesting that both genetics and the social environment contribute to rank acquisition in young geese. As juveniles typically form tight bonds with their parents, influences of the social environment may be more evident in juveniles than in older birds, which may explain why we found parental effects to be most pronounced at this life-history stage. Parental effects on behavior expressed later in life are possible through long-lasting consequences of nongenetic effects, but they may also represent a heritable genetic component of the behavior. The generally strong parental effects observed in this study support existing evidence that dominance-related behavior shows a heritable component (Craig et al. 1965; Boag 1982).

Interestingly though, parental effects were absent in adult singles and parental birds, that is, social classes that take the lowest and highest positions, respectively, in the dominance hierarchy of the flock. Social stress (at a low rank position) or fierce competition (at a high rank position) may be factors that constrain the full expression of the genetic predisposition for a certain behavior. At these positions in the flock hierarchy, the social environment may strongly define the frequency and the outcome of agonistic interactions. In future studies, multigenerational pedigrees could be employed to assess the relative contributions of genotype, parental environment, and social environment to individual differences in dominance and aggression.

\section{Life-history effects}

Our analyses confirmed the results of earlier studies in geese that reported males to be more aggressive and dominant than females (e.g., Boyd 1953; Black and Owen 1987; Poisbleau et al. 2006) and families to be dominant over pairs, whereas pairs tended to dominate single individuals (e.g., Lamprecht 1986; Gregoire and Ankney 1990; Kotrschal et al. 1993). As expected, social status was among the strongest determinants of dominance-related behaviors. Via increased agonistic success, social status may affect an individuals' access to critical resources like food. Indeed, studies in snow geese, Ross's geese (Chen rossii), and greylag geese found that individuals associated with their parents or offspring allocated more time to feeding than unassociated ones (Weiß and Kotrschal 2004; Jónsson and Afton 2009). Notably, the effects of sex and social status remained fairly constant in their magnitude and direction across the different life-history stages.

In contrast, effects of the raising history differed considerably between life-history stages. Juvenile hand-raised geese were less aggressive and lower ranking than goose-raised juveniles, whereas we observed the exact opposite in subadult geese. After fledging, hand-raised juveniles are no longer regularly accompanied by their human foster parents, thus lacking support from their most important social ally (Weiß and Kotrschal 2004). Possibly, hand-raised geese therefore learn to manage their relationships with other flock members without parental support at an earlier stage than goose-raised geese, which may explain why goose-raised individuals experienced a sharp drop in rank as subadults, but hand-raised subadults 
did not. Any disadvantages that hand-raised juveniles experience in direct contest with goose-raised geese may thereby be compensated at a later life-history stage. This may be one reason why adult hand-raised and goose-raised individuals do not differ in life-history traits, such as reproductive success (Hemetsberger et al. 2010). Furthermore, effects of the raising history were small or absent in adult individuals, which indicates that hand raising does not permanently affect aggressive behavior or dominance rank. Instead, our results suggest that hand raising shifted nongenetic parental effects to different life-history stages.

Age has been suggested to influence dominance and aggression, for example, in song sparrows, Melospiza melodia (Arcese and Smith 1985), and also in some goose studies but not in others (Lamprecht 1986; Black and Owen 1989; Stahl et al. 2001). In the present study, we detected a significant increase in dominance and aggression with age. However, these effects were comparatively small and suggest that age plays only a minor role in determining dominance and related behaviors in this goose flock.

Heterosexual pair bonds are the default in geese, but trios and homosexual pair bonds contribute up to $15 \%$ and $20 \%$ of the pair bonds, respectively (e.g., Huber and Martys 1993; Hemetsberger et al. 2010). Thus, these alliances are regularly adopted alternatives to the heterosexual pair bond. Homosexually paired males were previously described as being overtly aggressive and high ranking (Lorenz 1988; Huber and Martys 1993), but we found homosexually paired males to be less aggressive, specific, and dominant than heterosexual pairs or trios. Instead, our results support the idea that widowers or males unable to secure a female partner may engage in "homosocial" bonds as a "best of a bad job" tactic that allows them to outrank single individuals (Kotrschal et al. 2006). In trios, on the other hand, maintaining proximity between all social partners in a tight feeding situation may be more difficult than for pairs and may explain why trios are less aggressive and dominant than heterosexual pairs despite a larger unit size. The effects of other pair-bond characteristics (e.g., pair-bond duration, partner rank) on dominance and aggression were comparatively small.

Parents of secondary families were not only subordinate to parents of primary families but were also less aggressive, suggesting that family type influences investment in aggressive behaviors. Family size, on the other hand, had no noteworthy effect on the investigated behaviors, which is in contrast to several other studies. However, Loonen et al. (1999) proposed that the relationship of dominance rank and family size may depend on the amount of competition for resources. Larger families dominating small ones were described in studies in which food was scarce or patchily distributed (Gregoire and Ankney 1990; Loonen et al. 1999) but not in other studies, where food was abundant (Lamprecht 1986; Mulder et al. 1995). Due to supplemental feeding, food is abundant in our flock, and our results thus fit well to the idea proposed by Loonen et al. (1999).

\section{Seasonal effects}

Seasonal effects on dominance and aggression have received little attention (e.g., Tarvin and Woolfenden 1997), but our results highlight the importance of this environmental effect. Although seasonal effects were generally of moderate to small size, they showed the greatest variation across life-history stages in both aggression rates and dominance rank and thereby reflected the changing demands of individuals throughout the year. Pairs achieved the highest dominance rank during the mating season (February), when securing paternity and essential resources for breeding is of particular importance. Conse- quently, juveniles, subadults, and singles were least aggressive during the mating season, when agonistic pressure by paired individuals was highest. Parents, on the other hand, were most aggressive and dominant in summer, presumably to establish and demonstrate family rank to the reaggregated flock (Scheiber et al. 2009a). Parents won against fewest of their opponents during the mating season, which may reflect a reduction in parental investment as juveniles reach independence. In contrast to their parents, juveniles and subadults ranked highest during winter, when the (re-) establishment of dominance relations after the flock reunion in summer was finished and agonistic pressure from higher ranking flock members was lowest.

\section{Effects of flock structure}

The sex composition of the flock ranged from $35-50 \%$ females and had major effects on aggression rates, specificity, and on dominance rank in certain life-history stages. This indicates that sex not only influenced an individual's own behavior but also the social dynamics in the flock as a whole. Sex ratios in geese are rather poorly known, but as in our flock, they were male biased in a flock of bar-headed geese (Lamprecht 1987). Adult sex ratios are also frequently male biased in other waterfowl, likely due to differential survival, for example, during the breeding season (Blums and Mednis 1996; Kilpi et al. 2003). The present study showed that individual aggression rates and specificity generally increased with the number of males in the flock, independent of the initiating individual's own sex. This illustrates that the agonistic pressure experienced by individual members of a flock may depend considerably on the flock's sex ratio. Individuals that experience more aggression from flock members (because of more males present in the flock) will, in turn, also initiate more agonistic interactions themselves. Such a redirection of attacks against other flock members may serve to reduce tension, to reduce the rate of renewed attacks, or to restore access to resources (see Aureli et al. 1993). Unlike aggression rate and specificity, dominance rank was affected by the sex ratio of the flock only in juveniles and subadults. Arcese and Smith (1985) suggested that the accumulated experience of an individual in agonistic interactions is a key determinant of dominance. In a stable flock like ours, this may explain why sex ratio no longer had an influence on dominance rank in adults as individuals may have learned their own position in the dominance hierarchy as well as that of their flock members.

Estevez et al. (1997, 2003) hypothesized that with increasing group size, establishing dominance relationships with all group members becomes too costly and the social organization of domestic fowl (Gallus gallus domesticus) switches from a dominance hierarchy established through aggression to a tolerant social system characterized by low aggression. Our study provides only weak support for this. Geese were indeed less aggressive at larger flock sizes and if more families were present in the flock, but these effects were very small.

\section{CONCLUSIONS}

Our long-term study in greylag geese showed that a variety of intrinsic and extrinsic factors affected dominance and aggressive behavior to varying degrees. Parents and the sex ratio of the flock had particularly strong effects on juveniles and subadults, whereas these effects were often smaller or absent in adults. Our study thus demonstrates that the expression of agonistic behavior is a product of parental effects and a complex interplay of an individual's life-history stage and the social environment. 


\section{SUPPLEMENTARY MATERIAL}

Supplementary material can be found at http://www.beheco. oxfordjournals.org/.

\section{FUNDING}

Austrian "Fonds zur Förderung der wissenschaftlichen Forschung" (FWF Projects \# P10483-BIO, R30-B03, P18601-B17, and P18744-B03); Swiss "La Fondation Pierre Mercier pour la Science"; "Verein der Förderer der KLF;" and "Herzog von Cumberland Stiftung."

We are thankful to K. Mager for data collection in summer 2000 and J. Hemetsberger, I. Scheiber, and the various members of the goose team over the years for their efforts in monitoring of the flock. The editor and 2 anonymous referees provided helpful comments on the manuscript and our thanks go to Orlaith Fraser for correcting the English.

\section{REFERENCES}

Appleby MC. 1983. Competition in a red deer stag social group: rank, age and relatedness of opponents. Anim Behav. 31:913-918.

Arcese P, Smith JNM. 1985. Phenotypic correlates and ecological consequences of dominance in song sparrows. J Anim Ecol. 54: 817-830.

Aureli F, Veenema HC, Van Panthaleon CJ, van Hooff JARAM. 1993. Reconciliation, consolation, and redirection in Japanese macaques (Macaca fuscata). Behaviour. 124:1-21.

Black JM, Owen M. 1987. Determinants of social rank in goose flocks: acquisition of social rank in young geese. Behavior. 102:129-146.

Black JM, Owen M. 1989. Agonistic behavior in barnacle goose flocks: assessment, investment and reproductive success. Anim Behav. 37: 199-209.

Blums P, Mednis A. 1996. Secondary sex ratio in Anatinae. Auk. 113:505-511.

Boag DA. 1982. How dominance status of adult Japanese quail influences the viability and dominance status of their offspring. Can J Zool. 60:1885-1891.

Boag DA, Alway JH. 1980. Effect of social environment within the brood on dominance rank in gallinaceous birds (Tetraonidae and Phasianidae). Can J Zool. 58:44-49.

Boyd H. 1953. On encounters between wild white-fronted geese in winter flocks. Behavior. 5:85-129.

Brown JL. 1963. Dominance and social organization in the Steller jay. Condor. 65:460-484.

Buston P. 2003. Size and growth modification in clownfish. Nature. 424:145-146.

Clutton-Brock TH, Sheldon BC. 2010. The seven ages of Pan. Science. 327:1207-1208.

Côté SD. 2000. Dominance hierarchies in female mountain goats: stability, aggressiveness and determinants of rank. Behavior. 137: $1541-1566$

Craig JV, Ortman LL, Guhl AM. 1965. Genetic selection for social dominance ability in chickens. Anim Behav. 8:114-131.

Dunbar RIM. 1980. Determinants and evolutionary consequences of dominance among female Gelada baboons. Behav Ecol Sociobiol. 7:253-265.

Engh AL, Esch K, Smale L, Holekamp KE. 2000. Mechanisms of maternal rank "inheritance" in the spotted hyaena, Crocuta crocuta. Anim Behav. 60:323-332.

Estevez I, Keeling LJ, Newberry RC. 2003. Decreasing aggression with increasing group size in young domestic fowl. Appl Anim Behav Sci. 84:213-218.

Estevez I, Newberry RC, Arias de Reyna L. 1997. Broiler chickens: a tolerant social system? Etologia. 5:19-29.

Ficken MS, Weise CM, Popp JW. 1990. Dominance rank and resource access in winter flocks of black-capped chickadees. Wilson Bull. 102:623-633.

Galwey NW. 2006. Introduction to mixed modelling. Chichester (UK): Wiley.
Garamszegi LZ, Calhim S, Dochtermann N, Hegyi G, Hurd PL, Jorgensen C, Kutsukake N, Lajeunesse MJ, Pollard KA, Schielzeth H, et al. 2009. Changing philosophies and tools for statistical inferences in behavioral ecology. Behav Ecol. 20:1363-1375.

Gregoire PE, Ankney CD. 1990. Agonistic behavior and dominance relationships among lesser snow geese during winter and spring migration. Auk. 107:550-560.

Hemetsberger J. 2001. The demographic development of Konrad Lorenz' flock of greylag geese in Grünau/Austria since 1973. In: Kotrschal K, Müller G, Winkler H, editors. Konrad Lorenz und seine verhaltensbiologischen Konzepte aus heutiger Sicht. Fürth (Germany): Filander. p. 249-260.

Hemetsberger J, Scheiber IBR, Weiß BM, Frigerio D, Kotrschal K. 2010. Influence of socially involved hand-raising on life history and stress responses in greylag geese. Interact Stud. 11:380-395.

Hogstad O. 1987. It is expensive to be dominant. Auk. 104:33-336.

Horrocks J, Hunte W. 1983. Maternal rank and offspring rank in vervet monkeys: an appraisal of the mechanisms of rank acquisition. Anim Behav. 31:772-782.

Huber R, Martys M. 1993. Male-male pairs in greylag geese (Anser anser). J Ornithol. 134:155-164.

Jónsson JE, Afton AD. 2008. Lesser snow geese and Ross's geese form mixed flocks during winter but differ in family maintenance and social status. Wilson J Ornithol. 120:725-731.

Jónsson JE, Afton AD. 2009. Time budgets of snow geese Chen caerulescens and Ross's geese Chen rossii in mixed flocks: implications of body size, ambient temperature and family associations. Ibis. 151:134-144.

Kikkawa J. 1980. Winter survival in relation to dominance classes among silvereyes Zosterops lateralis chlorocephala of Heron Island, Great Barrier Reef. Ibis. 122:437-446.

Kikkawa J, Smith JNM, Prys-Jones R, Fisk P, Catterall C. 1986. Determinants of social dominance and inheritance of agonistic behavior in an island population of silvereyes, Zosterops lateralis. Behav Ecol Sociobiol. 19:165-169.

Kikkawa J, Wilson JM. 1983. Breeding and dominance among the Heron Island silvereyes Zosterops lateralis chlorocephala. Emu. 83: 181-198.

Kilpi M, Öst M, Lehikoinen A, Vattulainen A. 2003. Male sex bias in eiders Somateria mollissima during spring migration into the Gulf of Finland. Ornis Fenn. 80:137-142.

Kotrschal K, Hemetsberger J, Dittami J. 1993. Food exploitation by a winter flock of greylag geese: behavioral dynamics, competition and social status. Behav Ecol Sociobiol. 33:289-295.

Kotrschal K, Hemetsberger J, Weiß BM. 2006. Making the best of a bad situation: homosociality in male greylag geese. In: Sommer V, Vasey PL, editors. Homosexual behavior in animals: an evolutionary perspective. Cambridge (MA): Cambridge University Press. p. 45-76.

Kotrschal K, Hirschenhauser K, Möstl E. 1998. The relationship between social stress and dominance is seasonal in greylag geese. Anim Behav. 55:171-176.

Kotrschal K, Scheiber IBR, Hirschenhauser K. 2010. Individual performance in complex social systems: the greylag goose example. In: Kappeler P, editor. Animal behavior: evolution and mechanisms. Heidelberg (Germany): Springer Verlag. p. 121-148.

Kristiansen JN, Jarrett NS. 2002. Inter-specific competition between Greenland white-fronted geese Anser albifrons flavirostris and Canada geese Branta canadensis interior moulting in West Greenland: mechanisms and consequences. Ardea. 90:1-13.

Lamprecht J. 1986. Structure and causation of the dominance hierarchy in a flock of bar-headed geese (Anser indicus). Behavior. 96: 28-48.

Lamprecht J. 1987. Female reproductive strategies in bar-headed geese (Anser indicus): why are geese monogamous? Behav Ecol Sociobiol. 21:297-305.

Loonen MJJE, Bruinzeel LW, Black JM, Drent RH. 1999. The benefit of large broods in barnacle geese: a study using natural and experimental manipulations. J Anim Ecol. 68:753-768.

Lorenz K. 1988. Hier bin ich-wo bist Du? Ethologie der Graugans. München (Germany): Piper Verlag.

Madsen J. 1985. Habitat selection of farmland feeding geese in West Jutland, Denmark: an example of a niche shift. Ornis Scand. 16: $140-144$ 
Mulder RS, Williams TD, Cooke F. 1995. Dominance, brood size and foraging behavior during brood-rearing in the lesser snow goose: an experimental study. Condor. 97:99-106.

Poesel A, Kunc HP, Foerster K, Kempenaers B. 2006. Early birds are sexy: male age, dawn song and extrapair paternity in blue tits, Cyanistes (formerly Parus) caeruleus. Anim Behav. 72:531-538.

Poisbleau M, Fritz H, Valeix M, Perroi P-Y, Dalloyau S, Lambrechts MM. 2006. Social dominance correlates and family status in wintering dark-bellied brent geese, Branta bernicla bernicla. Anim Behav. 71:1351-1358.

Prop J, Deerenberg C. 1991. Spring staging in brent geese Branta bernicla: feeding constraints and the impact of diet on the accumulation of body reserves. Oecologia. 87:19-28.

Pusey A, Williams J, Goodall J. 1997. The influence of dominance rank on the reproductive success of female chimpanzees. Science. 277: 828-831.

Raveling DG. 1970. Dominance relationships and agonistic behavior of Canada geese in winter. Behavior. 37:291-319.

Richner H. 1989. Phenotypic correlates of dominance in carrion crows and their effects on access to food. Anim Behav. 38:606-612.
Sapolsky RM. 2005. The influence of social hierarchy on primate health. Science. 308:648-652.

Scheiber IBR, Kotrschal K, Weiß BM. 2009b. Benefits of family reunions: social support in secondary greylag goose families. Horm Behav. 55:133-138.

Scheiber IBR, Kotrschal K, Weiß BM. 2009a. Serial agonistic attacks by greylag goose families (Anser anser) against the same target. Anim Behav. 77:1211-1216.

Scheiber IBR, Weiß BM, Frigerio D, Kotrschal K. 2005. Active and passive social support in families of greylag geese (Anser anser). Behavior. 142:1535-1557.

Stahl J, Tolsma P, Loonen MJJ, Drent RH. 2001. Subordinates explore but dominants profit: resource competition in high Arctic barnacle goose flocks. Anim Behav. 61:257-264.

Tarvin KA, Woolfenden GE. 1997. Patterns of dominance and aggressive behavior in blue jays at a feeder. Condor. 99:434-444.

Weiß BM, Kotrschal K. 2004. Effects of passive social support in juvenile greylag geese (Anser anser): a study from fledging to adulthood. Ethology. 110:429-444. 